\title{
Functional magnetic resonance imaging of a parametric working memory task in schizophrenia: relationship with performance and effects of antipsychotic treatment
}

\author{
Ulrich Ettinger • Steven C. R. Williams • Dominic Fannon • Preethi Premkumar • \\ Elizabeth Kuipers • Hans-Jürgen Möller • Veena Kumari
}

Received: 30 July 2010 / Accepted: 17 January 2011 /Published online: 18 February 2011

(C) The Author(s) 2011. This article is published with open access at Springerlink.com

\begin{abstract}
Rationale Working memory dysfunction is frequently observed in schizophrenia. The neural mechanisms underlying this dysfunction remain unclear, with functional neuroimaging studies reporting increased, decreased or unchanged activation compared to controls.

Objectives We investigated the neural correlates of spatial working memory in schizophrenia with particular consideration of effects of antipsychotic treatment and relation to performance levels in the patient group.

Method We used functional magnetic resonance imaging and studied the blood-oxygen-level-dependent (BOLD)
\end{abstract}

Electronic supplementary material The online version of this article (doi:10.1007/s00213-011-2214-7) contains supplementary material, which is available to authorized users.

\section{U. Ettinger $(\bowtie) \cdot$ H.-J. Möller}

Department of Psychiatry, University of Munich,

Nussbaumstr. 7,

80336 Munich, Germany

e-mail: ulrich.ettinger@med.uni-muenchen.de

\section{U. Ettinger}

Department of Psychology, University of Munich,

Munich, Germany

\section{S. C. R. Williams}

Department of Neuroimaging, King's College London,

Institute of Psychiatry,

London, UK

D. Fannon

Division of Psychological Medicine, King's College London, Institute of Psychiatry,

London, UK

D. Fannon $\cdot$ P. Premkumar $\cdot$ E. Kuipers $\cdot$ V. Kumari Department of Psychology, King's College London, Institute of Psychiatry,

London, UK response of 45 schizophrenia outpatients and 19 healthy controls during a parametric spatial $n$-back task.

Results Performance in both groups deteriorated with increasing memory load (0-back, 1-back, 2-back), but the two groups did not significantly differ in performance overall or as a function of load. Patients produced stronger BOLD signal in occipital and lateral prefrontal cortex during task performance than controls. This difference increased with increasing working memory load in the prefrontal areas. We also found that in patients with good task performance, the BOLD response in left prefrontal cortex showed a stronger parametric increase with working memory load than in patients with poor performance. Second-generation antipsychotics were independently associated with left prefrontal BOLD increase in response to working memory load, whereas first-generation antipsychotics were associated with BOLD decrease with increasing load in this area.

Conclusions Together, these findings suggest that in schizophrenia patients, normal working memory task performance may be achieved through compensatory neural activity, especially in well-performing patients and in those treated with second-generation antipsychotics.

Keywords Schizophrenia - Spatial working memory · Functional brain imaging $\cdot$ Antipsychotics $\cdot$ Biomarker

\section{Introduction}

Working memory is defined as the capacity to mentally maintain and manipulate information over short time periods. As such, it is an important cognitive function underlying a range of behaviours and everyday tasks. Working memory deficits are frequently observed in schizophrenia (Barch 2005; Goldman-Rakic 1994; Park and Holzman 1992). The deficit has been observed at 
different illness stages and may be a predictor of clinical and functional outcome as well as a relevant target for treatments aimed at cognitive enhancement (Green and Nuechterlein 2004; Greenwood et al. 2005; Liddle 2000).

Basic neuroscience studies have shown that the dorsolateral prefrontal cortex (DLPFC) as well as parietal cortex and subcortical projection targets are of critical importance in working memory (Barch 2005; Gruber and von Cramon 2003). However, despite the often replicated observation of working memory impairments in schizophrenia, brain activation studies using functional magnetic resonance imaging (fMRI) have not yet reliably identified the neural circuits underlying this impairment. While initial studies observed reduced activation in frontal areas in patients (termed hypofrontality), other studies have reported increased activation or no significant differences between patients and controls (for review, see (Glahn et al. 2005; Linden 2009; Manoach 2003). A variety of factors such as task design and patient characteristics are likely to play a role, but task difficulty, subject performance levels, and pharmacological treatment status are considered to be particularly important factors.

Regarding the related factors of task difficulty and performance, it has been argued (Manoach 2003) and demonstrated empirically (Callicott et al. 2003; Potkin et al. 2009) that activation levels may fall along an inverted $U$ shape distribution reflecting task difficulty. This curve may differ between patients and healthy controls, with patients showing hyperactivations relative to controls at easier task difficulty levels (or when no behavioural impairments are seen) and hypoactivations at greater task difficulty (or when behavioural impairments are seen).

Regarding pharmacological treatment, the effects of antipsychotic medication have been addressed in a number of studies. These compounds have been shown to influence the blood-oxygen-level-dependent (BOLD) signal during performance of a variety of cognitive and behavioural paradigms. Specifically, a number of studies have shown normalisation of hypofunction in schizophrenia with second-generation (SGA) but not first-generation antipsychotics (FGA) across a range of neurocognitive paradigms (Braus et al. 1999; Honey et al. 1999; Jones et al. 2004; Kumari et al. 2007; Meisenzahl et al. 2006; Stephan et al. 2001; for review see Davis et al. 2005; Kumari and Cooke 2006). However, not all studies have observed this effect (Surguladze et al. 2007), and other work points to task dependence of treatment effects. For example, Schlagenhauf et al. (2008) found that switching from FGA to olanzapine led to increased BOLD in the left DLPFC during a 0-back attentional condition but not during a 2-back working memory condition, relative to baseline.

The aims of this study were to further explore the macroscopic neural circuits underlying working memory task performance in a large sample of schizophrenia patients. More work is needed in this area due to the inconsistencies in the literature and in order to build up the evidence base especially in relation to the not yet clarified issue of hypo- vs. hyperfrontality (Karch et al. 2009; Manoach 2003; Potkin et al. 2009; Schneider et al. 2007; Tan et al. 2005). Additionally, we aimed to examine differences in BOLD signal between patients treated with FGA compared to SGA. This aim is of relevance considering that working memory as well as the underlying BOLD response may represent promising treatment targets or biomarkers in drug development (Green and Nuechterlein 2004; Migo et al. 2011). To study working memory, we used a spatial, parametric $n$-back task. The $n$-back task is a widely used paradigm that may represent an intermediate phenotype reflecting genetic vulnerability to schizophrenia as well as a potential treatment target (Barch and Smith 2008; Egan et al. 2001; Linden 2009). The parametric design of the task allowed us to test for effects of working memory load on BOLD signal as well as its modulation by antipsychotic treatment.

\section{Method}

\section{Participants}

Patients with a DSM-IV diagnosis of schizophrenia (American Psychiatric Association 1994) were recruited from the South London and Maudsley catchment area. Patients were (ii) if treated, on stable doses of antipsychotics for $\geq 2$ years and on their present antipsychotic for $>3$ months, (iii) in a stable phase of the illness with stable symptoms for at least 3 months, (iv) living in the community or long stay/rehabilitation wards, and (v) free of any co-morbid psychiatric diagnosis. Symptom severity was rated using the Positive and Negative Syndrome Scale (PANSS; Kay et al. 1987).

Healthy controls were recruited via local advertisements from the same geographical area as the patients. Controls were required to be free from past or current drug abuse and were screened by an experienced psychiatrist to exclude current psychiatric conditions using the Structured Clinical Interview for DSM Axis I Disorders (First et al. 1996).

For both groups, only right-handed participants were included. An additional inclusion criterion for all participants was the absence of a history of neurological conditions or head injury. Participants provided written informed consent, and the study had research ethics committee permission.

\section{fMRI task}

The task (see Kumari et al. 2009) was a parametric spatial $n$-back paradigm that involved monitoring locations of dots within a diamond-shaped box on the screen at a given delay 
from the original occurrence (0-back, 1-back, or 2-back). Participants viewed the paradigm projected onto a screen through a prismatic mirror. They were required to press the button on every trial, using the right thumb, corresponding to the correct location of the 0-back, 1-back, or 2-back stimulus (location of dots random). The level of chance performance was $25 \%$.

Each condition (0-back, 1-back, 2-back) was presented five times in 30-s blocks in pseudo-random order, controlling for order effects. Each block contained 15 stimulus presentations (stimulus duration $=450 \mathrm{~ms}$, interstimulusinterval $=1,500 \mathrm{~ms}$ ) and began with a $750-\mathrm{ms}$ text delay allowing the participants to notice a change in task demand/ condition. There were 15-s rest blocks (presentation of the word "Rest" on the screen) between active blocks and following the last active block (thus, there was a total of 15 rest blocks). The experiment lasted $11.25 \mathrm{~min}$. Participants were requested to abstain from alcohol for at least $24 \mathrm{~h}$ before testing and underwent task familiarisation before scanning.

\section{fMRI data acquisition}

Echoplanar MR images of the whole brain were acquired using a Signa scanner (General Electric, Milwaukee, Wisconsin) at 1.5-Tesla field strength. In each of 16 nearaxial noncontiguous planes parallel to the intercommissural plane, $225 \mathrm{~T} 2 *$-weighted MR images depicting BOLD contrast were acquired over the experiment with echo time $(\mathrm{TE})=40 \mathrm{~ms}$, repetition time $(\mathrm{TR})=3,000 \mathrm{~ms}$, field of view $(\mathrm{FOV})=240 \mathrm{~mm}$, in-plane resolution $=3.75 \times 3.75 \mathrm{~mm}$, slice thickness $=7.0 \mathrm{~mm}$, and interslice gap $=0.7 \mathrm{~mm}$.

\section{$\mathrm{N}$-back performance data analysis}

The key-dependent variable measuring the success of $n$-back task performance was the percentage of correct responses. Additionally, we investigated the percentage of omission errors (failures to respond) and the latency of correct responses (in milliseconds). Data were analysed in SPSS Release 15.0.0 using repeated measures analysis of variance (ANOVA) with group (patients, controls) as betweensubjects factor and load (0-back, 1-back, 2-back) as withinsubjects factor to assess main and interaction effects. Eta squared $\left(\eta_{\mathrm{p}}{ }^{2}\right)$ is given as a measure of effect size.

\section{fMRI data analysis}

fMRI data analysis was carried out using Statistical Parametric Mapping software (SPM5; http://www.fil.ion. ucl.ac.uk/spm) running in Matlab R2008a (The MathWorks Inc.). Images were first motion corrected, spatially transformed (MNI template), smoothed $(8 \mathrm{~mm}$ full-width-at- half-maximum Gaussian filter). A high-pass filter with a cut-off of $128 \mathrm{~s}$ was applied.

Data were analysed within the framework of the general linear model implemented in SPM5. At the single-subject level contrast maps of each of the three conditions (0-back, 1-back, 2-back) were created with rest as implicit baseline by modelling, at each voxel, each condition using a boxcar function which incorporates the delay inherent in the hemodynamic response. Motion parameters obtained from the realignment pre-processing step were included as covariates at this stage. The resulting maps were entered into a random-effects procedure at the second level to investigate the main effect of group (patients, controls), the main effect of load (0-back, 1-back, 2-back), and the groupby-load interaction. The threshold of significance for these analyses was set at $p=0.05$ (corrected cluster level) with a height threshold of $p<0.001$ at the voxel level.

The group-by-load interaction effect was considered the first key result in this study; viz. differences between patients and controls in the brain functional response to parametric working memory increase. Therefore, while subsequent analyses of performance and treatment effects within the patient group were carried out first using an anatomically unconstrained whole-brain voxelwise method in SPM5, any such analyses were also repeated using a regions of interest (ROI) approach. ROIs were created by extracting the signal (using the MarsBaR toolbox implemented in SPM5; Brett et al. 2002) from clusters that showed a significant group-byload interaction. Statistical analyses of these ROIs in relation to performance and treatment were carried out using ANOVA in SPSS 15.0.0 as described below; these analyses thus allowed addressing the question of how areas in which patients differ from controls as a function of processing demands are associated with task performance and antipsychotic treatment within the patient group.

In order to investigate the role of performance levels on BOLD signal within the patient group, a performance (high, low) variable was created by splitting the patient group into those with high and those with low overall percentage of correct responses along the median of the patient group (the subject with the median score was excluded). These two patient groups were first compared to each other in clinical, demographic, and performance data. The two groups were then compared to each other in BOLD data, first across the entire brain in SPM5 and then restricted to ROIs using ANOVA in SPSS with performance (high, low) as betweensubjects factor and load (0-back, 1-back, 2-back) as withinsubjects factor. The effect size measure Eta squared $\left(\eta_{\mathrm{p}}{ }^{2}\right)$ is given for these effects.

In order to assess effects of antipsychotic treatment on task performance and BOLD response, the patient sample was split into those treated with FGA $(N=6)$ and those treated with SGA $(N=38)$ antipsychotics (one patient was 
unmedicated at the time of fMRI scanning and was excluded for the purpose of this analysis). These two groups were then compared to each other in clinical, demographic, and performance data. The two groups were also compared to each other in BOLD data, first across the entire brain in SPM5 and then restricted to ROIs using ANOVA in SPSS with treatment (FGA, SGA) as betweensubjects factor and load (0-back, 1-back, 2-back) as withinsubjects factor. Eta squared $\left(\eta_{\mathrm{p}}{ }^{2}\right)$ is again given.

Finally, the relationship between BOLD and symptom severity (PANSS) was investigated in the patient sample (first across all voxels in the whole brain in SPM5 and subsequently in the extracted ROIs in SPSS).

MNI coordinates of peaks within significant clusters were transformed to Talairach coordinates using a non-linear algorithm (see http://imaging.mrc-cbu.cam.ac.uk/imaging/ MniTalairach). The atlas of Talairach and Tournoux (1988) was used to identify anatomic labels and Brodmann areas (BA).

\section{Results}

\section{Sample description}

A total of 64 participants completed the study, consisting of 45 patients with schizophrenia and 19 controls. Demographic and clinical data are summarised in Table 1. The patient and control groups did not differ significantly on demographic variables with the exception of years spent in full-time education, which may be expected given that schizophrenia is commonly associated with lower than expected educational achievement (Green 2001; see also Surguladze et al. 2007).

The majority of patients $(N=38)$ were treated with SGA (clozapine $=10$, risperidone $=7$, olanzapine $=16$, amisulpride $=2$, aripiprazole $=3$ ), six patients were treated with FGA (haloperidol $=1$, flupentixol $=2$, sulpiride $=2$, chlorpromazine $=1$ ), and one patient was untreated at time of fMRI testing.

A number of patients were co-medicated with anticholinergic, benzodiazepine, mood stabilising, or antidepressant compounds. Anticholinergic compounds were administered to three patients on FGA and to two patients on SGA. Benzodiazepines were administered to four patients on SGA. Mood stabilisers were administered to one patient on FGA and eight patients on SGA. Antidepressants were administered to two patients on FGA and 15 patients on SGA.

\section{Working memory task performance}

Working memory task performance data are shown in Table 2 . The patient and control groups did not significantly differ in percent correct trials $\left(F[1,62]=2.17, p=0.15, \eta_{\mathrm{p}}{ }^{2}=\right.$ $0.03)$. There was an effect of load on percent correct trials $(F$ $\left.[2,124]=73.47, p<0.001, \eta_{\mathrm{p}}{ }^{2}=0.54\right)$, indicating fewer correct responses with increasing load, but no group-by-load interaction $\left(F[2,124]=1.27, p=0.29, \eta_{\mathrm{p}}^{2}=0.02\right)$.

The groups also did not significantly differ in the latency to correct trials $\left(F[1,61]=1.00, p=0.32, \eta_{\mathrm{p}}{ }^{2}=0.02\right)$. As with percent correct responses, there was an effect of load on latency $\left(F[2,122]=17.11, p<0.001, \eta_{\mathrm{p}}{ }^{2}=0.22\right)$, indicating longer latencies with increasing load, but no group-by-load interaction $\left(F[2,122]=0.80, p=0.45, \eta_{\mathrm{p}}^{2}=0.01\right)$.

Table 1 Demographic and clinical data

\begin{tabular}{llll}
\hline & Patients $(N=45)$ & Controls $(N=19)$ & Group comparison \\
\hline Age (years) & $37.33(8.19)$ & $33.32(9.21)$ & $t=-1.73, \mathrm{df}=62, p=0.09$ \\
Gender $(N$ male/ $N$ female) & $35 / 10$ & $12 / 7$ & $\chi^{2}=1.46, \mathrm{df}=1, p=0.23$ \\
Ethnicity $(N$ Caucasian/ $N$ other) & $19 / 26$ & $12 / 7$ & $\chi^{2}=2.34, \mathrm{df}=1, p=0.13$ \\
Years of education & $13.36(2.33)$ & $14.95(2.92)$ & $t=2.32, \mathrm{df}=62, p=0.02$ \\
Parental SES & $2.58(1.08)$ & $2.26(1.20)$ & $Z=-1.06, p=0.29$ \\
Duration of illness (years) & $13.49(9.78)$ & - & - \\
Age of onset (years) & $23.84(6.58)$ & - & - \\
Antipsychotic treatment $(\mathrm{N}$ SGA/N FGA) & $38 / 6^{\mathrm{a}}$ & - & - \\
PANSS positive symptoms & $16.18(4.72)$ & - & - \\
PANSS negative symptoms & $17.67(4.48)$ & - & - \\
PANSS general psychopathology & $32.49(6.01)$ & - & -
\end{tabular}

Data represent means (and standard deviations) unless indicated otherwise. Socio-economic status (SES) is measured in professional achievement from 1 (professional) to 4 (manual). All participants were right-handed

PANSS positive and negative syndrome scale, $S G A$ second-generation antipsychotics, $F G A$ first-generation antipsychotics

${ }^{\text {a }}$ One patient was untreated at the time of fMRI scanning 
Table $2 \mathrm{~N}$-back task performance data by group

\begin{tabular}{lcc}
\hline & Patients $(N=45)$ & Controls $(N=19)$ \\
\hline 0-back correct responses & $84.89(19.15)$ & $87.51(13.91)$ \\
1-back correct responses & $62.10(28.92)$ & $74.89(22.42)$ \\
2-back correct responses & $43.18(26.54)$ & $51.58(23.19)$ \\
0-back omissions & $8.03(9.62)$ & $8.56(9.26)$ \\
1-back omissions & $15.75(16.23)$ & $14.81(10.96)$ \\
2-back omissions & $27.49(20.92)$ & $29.72(18.15)$ \\
0-back latency & $233.95(130.96)$ & $191.36(145.83)$ \\
1-back latency & $296.41(210.07)$ & $293.70(145.88)$ \\
2-back latency & $490.24(353.46)$ & $390.63(213.42)$ \\
\hline
\end{tabular}

Data represent means. Standard deviations are given in parentheses. Correct responses and omissions are given in percent; latency is given in milliseconds.

Similarly, there was an effect of load on the percentage of omission errors $\left(F[2,124]=38.24, p<0.001, \eta_{\mathrm{p}}{ }^{2}=0.38\right)$, indicating more omission errors with increasing load, but no effect of group $\left(F[1,62]=0.04, p=0.85, \eta_{\mathrm{p}}{ }^{2}=0.001\right)$ or group-by-load interaction $(F[2,124]=0.23, p=0.80$, $\left.\eta_{\mathrm{p}}{ }^{2}=0.004\right)$.

\section{BOLD response by group and load}

Both groups activated an extensive fronto-parieto-striatothalamo-cerebellar network during performance of the $n$ back task. Figure 1 shows the activation of the combined group as a function of load (i.e., increase in BOLD signal with increasing working memory load); the main effect of task (across conditions of load) in the combined sample gave a very similar result.

The main effect of group (across conditions of load) revealed three clusters of increased activation $(p<0.05$ corrected cluster level) in patients relative to controls: (1) occipital cortex (BA17; Talairach coordinates of peak voxel, $x=-20, y=-88, z=-9 ; 857$ voxels; $Z=4.39$ ) extending into the cerebellum; (2) left middle frontal gyrus (BA9; $x=-46, y=15, z=36 ; 1,913$ voxels; $Z=4.26$ ) extending into pre- and postcentral gyrus; and (3) right inferior frontal gyrus (BA45/47; $x=46, y=18, z=18 ; 854$ voxels; $Z=4.06$ ) extending into right middle frontal gyrus. In contrast, there were no significant increases in controls relative to patients at the corrected or uncorrected cluster level and there were no significant voxels at the chosen height threshold $(p<0.001)$.

The group-by-load interaction (Fig. 2) revealed two significant clusters ( $p<0.05$ corrected cluster level): (1) left middle frontal gyrus (BA9; $x=-46, y=17, z=36$; 2,032 voxels; $Z=4.43$ ) and (2) right inferior frontal gyrus (BA45/ $47 ; x=46, y=18, z=18 ; 749$ voxels; $Z=3.94$ ) extending into right middle frontal gyrus. A third cluster with a peak in the occipital cortex (BA17; $x=-22, y=-86, z=-11 ; 535$ voxels; $Z=4.10$ ) showed the same pattern but narrowly missed the level of statistical significance $(p=0.054$, corrected cluster level). These clusters showed stronger increases across load in patients than in controls. On the other hand, there were no significant clusters showing stronger increases across load in controls than in patients at the corrected or uncorrected cluster level, and there were no significant voxels at the chosen height threshold $(p<0.001)$.

The prefrontal clusters that emerged in this interaction analysis appeared not to be part of the activation seen in response to load across both groups (Fig. 1) but appeared to be neighbouring it. In order to verify this, the group-byload interaction contrast was masked with the contrast image resulting from the main effect of load; the two prefrontal areas from the interaction analysis also emerged in this masked analysis, indicating that these are voxels that show an additional increase with load in the patient group but not in the controls and not in the combined group.

To better understand the origin of these group-by-load interaction effects, the mean BOLD signal in each of the two frontal clusters that showed a significant interaction effect was extracted as described above and repeated measures ANOVAs with the within-subjects factor of load were run in SPSS separately for the two groups. In the left prefrontal cluster, we found a linear increase in BOLD as a

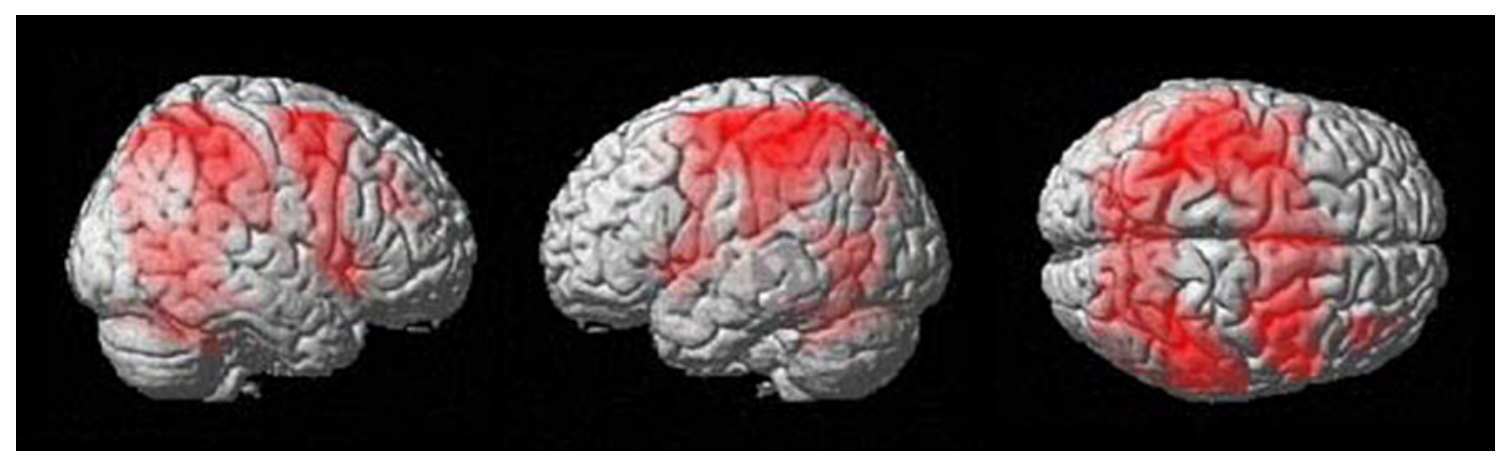

Fig. 1 Main effect of load on BOLD across groups. The figure depicts significant BOLD response as a function of load on the $n$-back working memory task across both groups $(p<0.05$, FWE corrected voxel level) 


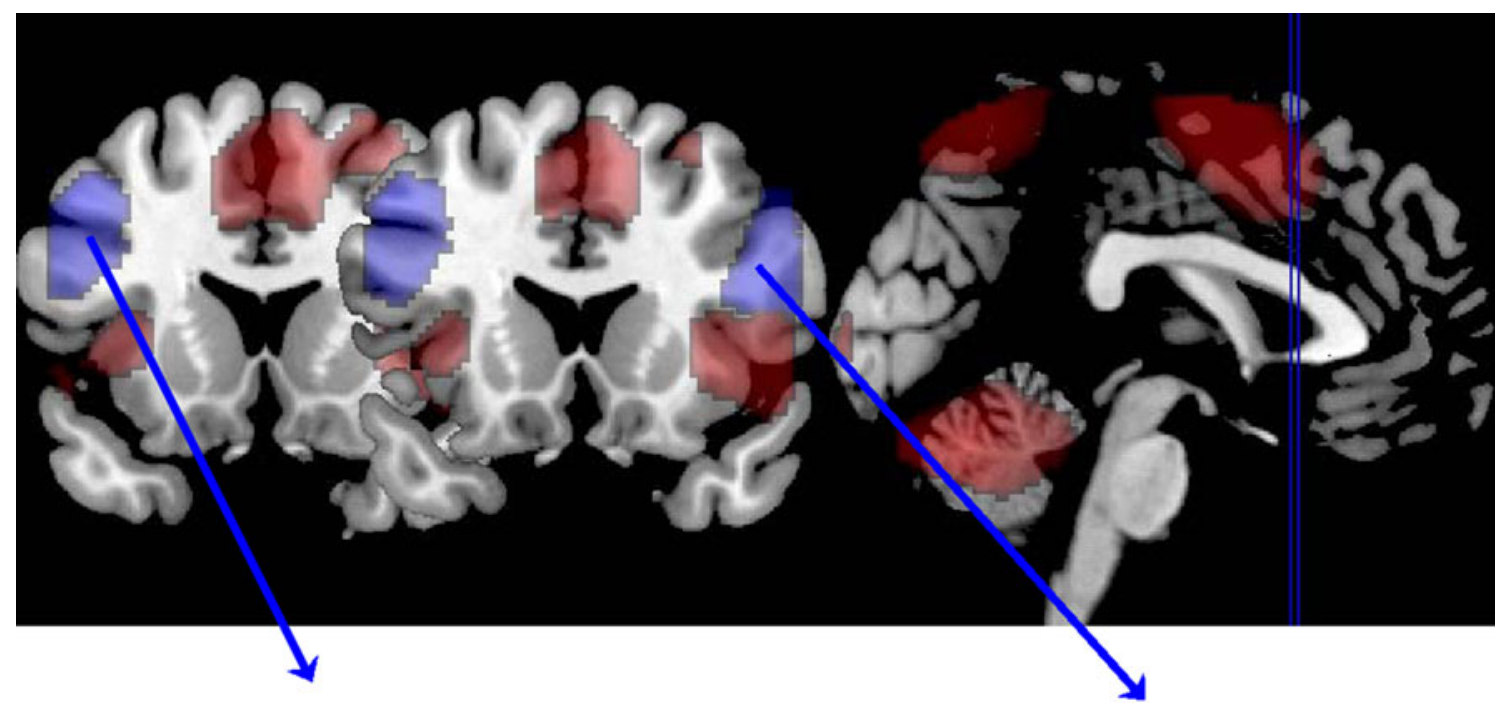

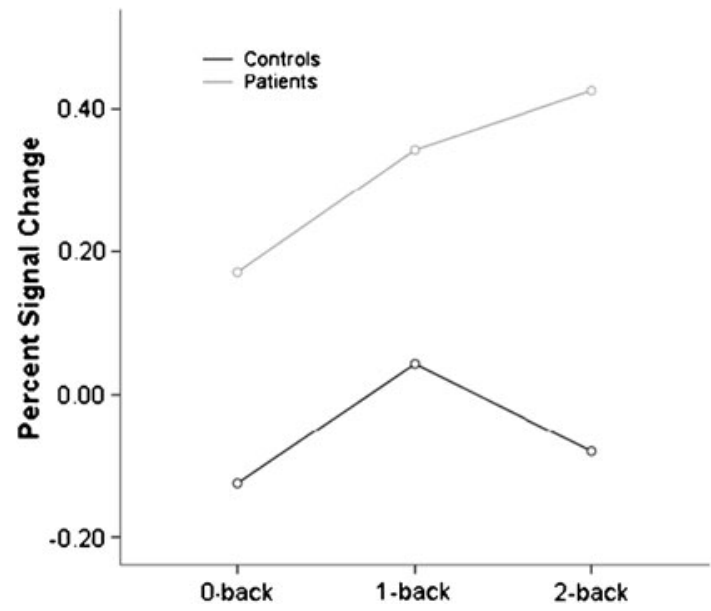

Fig. 2 Group-by-load interactions in BOLD response. The upper part of the figure depicts in red the areas that show a significant main effect of load and in blue the two prefrontal areas that show a significant group-by-load interaction. The selection of the two coronal

function of load in the patient group $(F[2,88]=6.65, p=$ $\left.0.002, \eta_{\mathrm{p}}{ }^{2}=0.13\right)$ but not in the controls $(F[2,36]=0.35$, $\left.p=0.71, \eta_{\mathrm{p}}^{2}=0.02\right)$. Similarly, in the right prefrontal cluster, there was a linear increase in BOLD as a function of load in the patient group $(F[2,88]=8.26, p=0.001$, $\left.\eta_{\mathrm{p}}{ }^{2}=0.16\right)$ but not in the controls $(F[2,36]=0.77, p=$ $\left.0.47, \eta_{\mathrm{p}}^{2}=0.04\right)$

Taken together, this pattern indicates that patients showed a significantly greater BOLD increase in response to working memory load than controls in lateral prefrontal cortical areas that did not show a significant main effect of load.

Association of BOLD with performance level in the patient group

In keeping with the analysis plan, the patient group was split into those with high and low performance along the

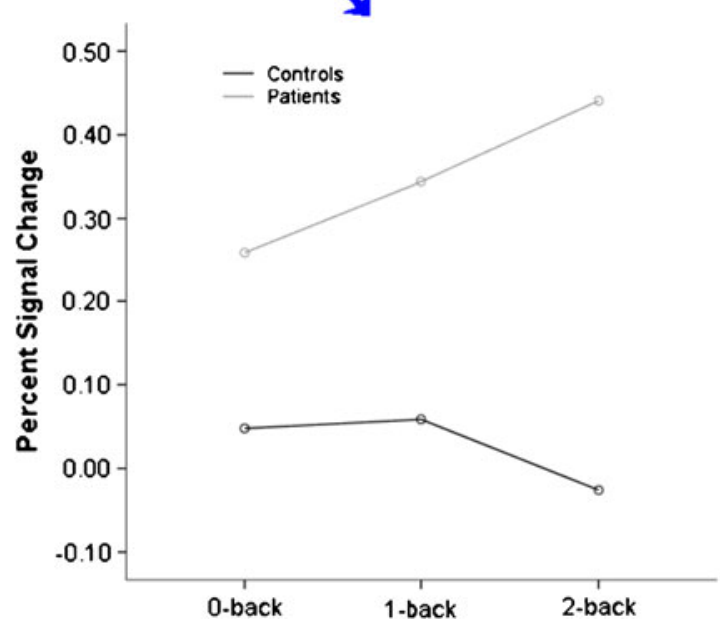

slices corresponds to the Talairach $y$ coordinates of the peak voxel for the right $(y=18)$ and left $(y=17)$ PFC clusters, respectively. The lower parts of the figure depict the nature of the interaction effects separately for left and right PFC

patient group's median $(62.49 \%)$, yielding two groups of $N=$ 22 each (the subject with the median score was excluded). $N$-back data of the two groups are summarised in Table 3. Importantly, the high- and low-performing patient groups did not differ from each other in any demographic or clinical variables (all $p>0.05$ ). However, the two patient groups differed significantly from each other in percent correct response at each level of load (all $F>11.88, p<0.002$ ), as expected. They also differed with regards to omission errors (all $F>6.82, p<0.02$ ) but not in reaction time (all $F<1.85, p>0.18$ ).

At the level of BOLD, each patient subgroup showed similar activation increases in response to load as the combined group (see Online Resource 1). An anatomically unconstrained voxelwise comparison between the two patient groups did not find a main effect of performance and there was no performance-by-load interaction (at $p=$ 0.05 , corrected cluster level). A comparison of the 
Table 3 Performance data by patient subgroups

\begin{tabular}{|c|c|c|c|c|}
\hline & \multicolumn{2}{|c|}{ Performance subgroups } & \multicolumn{2}{|l|}{ Treatment subgroups } \\
\hline & High $(N=22)$ & Low $(N=22)$ & First-generation $(N=6)$ & Second-generation $(N=38)$ \\
\hline 0-back correct responses & $93.52(5.77)$ & $75.64(23.63)$ & $89.11(9.22)$ & $84.07(20.50)$ \\
\hline 1-back correct responses & $85.07(10.73)$ & $39.87(23.55)$ & $60.48(31.03)$ & $63.27(28.81)$ \\
\hline 2-back correct responses & $62.17(23.96)$ & $24.20(12.11)$ & $43.59(30.75)$ & $43.77(26.32)$ \\
\hline 0-back omissions & $4.61(4.15)$ & $11.76(12.16)$ & $8.00(6.14)$ & $8.07(10.24)$ \\
\hline 1-back omissions & $7.34(6.11)$ & $24.74(18.65)$ & $24.05(21.22)$ & $14.21(15.39)$ \\
\hline 2-back omissions & $18.88(17.24)$ & $36.92(20.80)$ & $37.95(24.36)$ & $25.26(20.11)$ \\
\hline 0-back latency & $220.16(103.08)$ & $254.48(153.69)$ & $268.35(129.35)$ & $218.62(117.80)$ \\
\hline 1-back latency & $252.88(144.17)$ & $303.00(195.38)$ & $442.97(228.72)$ & $264.07(193.76)$ \\
\hline 2-back latency & $403.39(267.89)$ & $537.96(380.03)$ & $460.99(237.67)$ & $488.19(372.91)$ \\
\hline
\end{tabular}

Data represent means. Standard deviations are given in parentheses. Correct responses and omissions are given in percent; latency is given in msec. The performance and treatment groups were not significantly associated (see "Results").

prefrontal clusters that had showed group-by-load interactions above similarly did not yield significant main effects of performance for either cluster (both $p>0.79$ ). However, there was a significant performance-by-load interaction for the left $\left(F[2,84]=4.09, p=0.02, \eta_{\mathrm{p}}^{2}=0.09\right)$ but not right $\left(p=0.24, \eta_{\mathrm{p}}{ }^{2}=0.03\right)$ cluster. In order to better understand this interaction, within-subject ANOVAs of load in the left prefrontal cluster were calculated separately for the two performance groups. These showed that the high- $(F[2,42]=$ 9.63, $\left.p<0.001, \eta_{\mathrm{p}}{ }^{2}=0.31\right)$ but not the low-performing patients $\left(p=0.83, \eta_{\mathrm{p}}^{2}=0.009\right)$ showed a statistically significant linear increase in BOLD with load (Fig. 3).

\section{Effects of antipsychotic treatment}

The split according to treatment yielded two groups of patients, those treated with FGA $(N=6)$ and those treated with SGA $(N=38)$ antipsychotics $(N=1$ excluded $) . N$ -

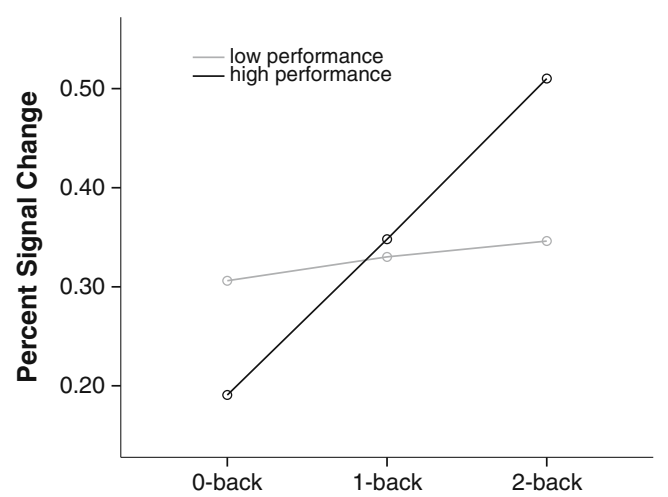

Fig. 3 Performance-by-load interaction in BOLD response. The figure shows the performance-by-load interaction for the left PFC. The patient sample is split along the median correct response rate into those with high performance $(N=22)$ and those with low performance $(N=22)$. The $x$ axis shows the three conditions of the $n$-back working memory task back performance data of the treatment groups are summarised in Table 3. There were no significant associations of treatment with any demographic variables, clinical variables, or anticholinergic, antidepressant, mood stabiliser, or benzodiazepine treatment (all $p>0.34$ ), and the treatment factor did not significantly interact with the performance variable $\left(\chi^{2}=0.89\right.$, df $\left.=1, p=0.35\right)$ : in the SGA group, there were 17 low- and 20 high-performing patients, and in the FGA group, there were four low- and two high-performing patients. There were no main effects of treatment (all $p>0.18$ ) or treatment-by-load interactions (all $p>0.22$ ) on $n$-back task performance; therefore, any treatment effects on BOLD reported below are considered to be independent of performance effects.

At the level of BOLD, an unrestricted voxelwise analysis did not yield any significant differences between the two treatment groups or treatment-by-load interactions (all $p>$ 0.05 , corrected cluster level). When considering the extracted prefrontal ROIs, no main effects of treatment were seen in either cluster (both $p>0.51$ ). However, there was a significant treatment-by-load interaction $(F[2,84]=$ 5.16, $\left.p=0.008, \eta_{\mathrm{p}}{ }^{2}=0.11\right)$ in the left prefrontal cluster. This interaction (Fig. 4) indicated that patients treated with SGA showed an increase in BOLD as a function of load, whereas the patients treated with FGA showed a reduction. The interaction effect appeared similar in the right prefrontal cluster but did not reach statistical significance $(p=0.13)$. A further split of the SGA into those patients treated with clozapine $(N=10)$, risperidone $(N=7)$, and olanzapine $(N=16)$ did not yield any significant group or interaction effects for the prefrontal ROIs. Therefore, the SGA group as a whole showed a stronger parametric left prefrontal BOLD increase than the FGA group, with no significant differences detected between different SGA compounds. 


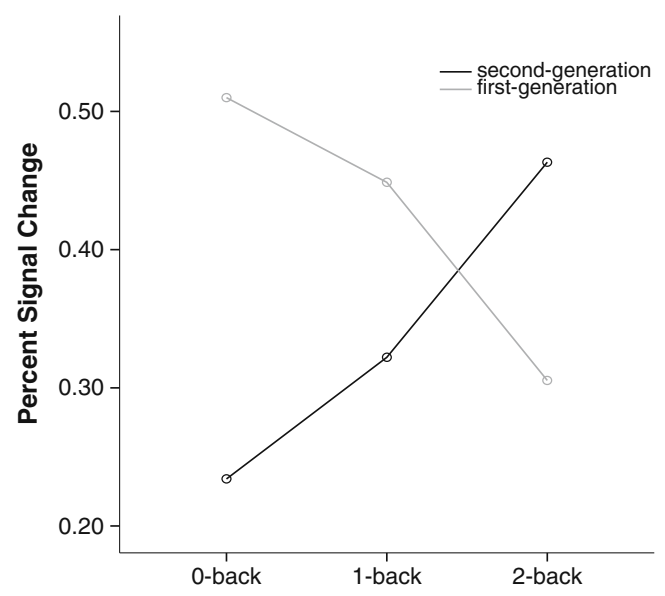

Fig. 4 Treatment-by-load interaction in BOLD response. The figure shows the treatment-by-load interaction for the left PFC. The patient sample is split into those treated with first-generation antipsychotics $(N=$ $6)$ and those treated with second-generation antipsychotics $(N=38)$. The $x$ axis shows the three conditions of the $n$-back working memory task

Association of BOLD with symptom severity

There were no significant correlations of BOLD from the combined $n$-back activations with PANSS total or subscale scores (all $p>0.05$, corrected cluster level). Similarly, there were no correlations between the extracted ROIs and PANSS symptom scores (all $p>0.06$ ).

\section{Discussion}

This study used fMRI to investigate the BOLD response during a parametric spatial working memory task in stable outpatients with schizophrenia and healthy control participants. It was found that (1) patients and controls did not differ significantly in task performance, (2) both groups showed deterioration in performance and increase in BOLD signal in response to increasing working memory load, (3) the patient group as a whole showed stronger BOLD signal in occipital and prefrontal cortical areas additional to prefrontal areas activated in both groups, (3) this difference increased with increasing working memory load in prefrontal areas, (4) patients with good performance showed greater left prefrontal BOLD increase in response to increasing working memory load than patients with poor performance, and (5) secondgeneration antipsychotics (SGA) were associated with a greater parametric increase in left prefrontal BOLD in response to increasing working memory load than first-generation antipsychotics (FGA), independent of the performance effects.

\section{Patient-control differences}

Working memory deficits in schizophrenia have often been demonstrated and it is important to understand this impairment in relation to the pathophysiology, genetics, and treatment of schizophrenia (Barch and Smith 2008; Goldman-Rakic 1994). However, there is still considerable lack of clarity concerning the neural mechanisms that mediate these cognitive impairments. Given the importance of the neurophysiological level of analysis in genetic (Meyer-Lindenberg and Weinberger 2006) and pharmacological (Migo et al. 2011) studies of schizophrenia, the aim of this study was to further address this issue.

Patients and controls did not differ in working memory performance in this study (see also Tan et al. 2005). At the level of BOLD, it was found that patients with schizophrenia displayed significantly stronger activation than controls in lateral prefrontal cortex and left occipital cortex (extending into the cerebellum) during task performance. Additionally, the group difference in prefrontal cortex became stronger with increasing load. The prefrontal cortex clusters were located to superior and middle frontal gyrus as well as medial prefrontal cortex. These prefrontal hyperactivations in patients appeared to be recruited in addition to prefrontal areas that were activated in the main effect of working memory load in both groups.

Previous brain activation studies of schizophrenia patients have variably shown increased, decreased, or comparable frontal activation levels in patients. These discrepancies have been attributed to a number of variables including performance and task difficulty (Callicott et al. 2003; Manoach 2003; Potkin et al. 2009). As outlined in detail elsewhere (Linden 2009), the pattern of schizophreniacontrol differences in BOLD signal and task performance can take on different forms, indicating qualitatively different, reduced, compensatory, aberrant, or inefficient neural response. The results of the current study indicate that areas activated by both groups are activated to a similar extent; however, patients additionally activated lateral prefrontal areas, an effect that became more pronounced with increasing task demands. Bearing in mind that the patients have psychotic symptoms and general clinical impairments, this pattern suggests the operation of compensatory neural mechanisms that led to overall successful task performance when compared to healthy controls. A number of previous studies have similarly observed compensatory BOLD increases on the background of comparable task performance in schizophrenia patients (Karch et al. 2009; Lee et al. 2008; Potkin et al. 2009; Royer et al. 2009; Tan et al. 2005; van Raalten et al. 2008; see also meta-analysis by Minzenberg et al. 2009). However, while the patients' recruitment of additional areas in our study may represent a behaviourally successful compensatory mechanism, it is also neurally inefficient as it requires additional resources in order to achieve a comparable performance levels as the controls. Of interest, it should be noted that compensatory hyperactivations have been observed not only in schizophrenia but also 
in other psychiatric conditions such as obsessive-compulsive disorder (Henseler et al. 2008).

Performance effects in patients

The left prefrontal BOLD increases in response to working memory load were more pronounced in patients with high levels of performance than in patients with low performance levels. The finding of performance-related activation levels in the patients is in part compatible with existing models which attribute group differences in BOLD to variation in task performance (Callicott et al. 2003; Manoach 2003). These models suggested that relatively unimpaired performance may be associated with increased BOLD signal, whereas lower performance may be associated with reductions in BOLD, e.g., hypofrontality. Given that high-performing patients in this study showed a stronger increase in activation with increasing task demands than low-performing patients it may be speculated that this may perhaps be compatible with low-performing patients not being able to further increase their BOLD beyond a certain level of task difficulty, which could have resulted in (or stemmed from) their lower working memory capacity. Relating this pattern to the inverted $U$ curves depicted by Manoach (2003) and Callicott et al. (2003), it may be surmised that low-performing patients are already at the top or on the downward slope of the inverted $U$.

\section{Treatment effects in patients}

The cross-sectional treatment effects observed here are compatible with some previous evidence (see "Introduction"). Patients treated with SGA showed load-related increases in left prefrontal BOLD, whereas those on FGA did not; it is important to note that this effect represents an interaction effect of working memory load and treatment but not a treatment main effect. Importantly, the FGA and SGA patient groups did not differ in demographic, clinical, or performance variables. An analysis of individual SGA compounds (clozapine, risperidone, olanzapine) did not yield any differences amongst these. As stated earlier, a number of pharmacological fMRI studies of antipsychotic compounds in schizophrenia have shown stronger BOLD signal with SGA compared to FGA. The strongest evidence for this effect comes from longitudinal studies which have shown that SGA improve cortical activations, thus normalising previous hypoactivations (review, Davis et al. 2005; Kumari and Cooke 2006; Migo et al. 2011). The present study observed this effect in a cross-sectional design. The effect was seen despite the small number of patients treated with FGA, a factor that can be attributed to current guidelines in clinical practice.

However, it should be noted that not all previous studies have found this effect. For example, Surguladze et al.
(2007) observed that patients on FGA showed an increase in ventromedial prefrontal BOLD as a function of working memory load, whereas patients treated with risperidone and healthy controls did not. Conversely, a lateral ventral prefrontal area studied by Surguladze et al. (2007) showed an increase with load only in controls but not in FGA- or risperidone-treated patients. An important difference between their study and ours is that in Surguladze et al. (2007), patients treated with FGA displayed significantly impaired task performance relative to risperidone-treated patients, whereas in our study, there were no significant drug effects on the level of task performance. Furthermore, there is evidence for relative functional specificity within prefrontal cortices. For example, studies have shown bilaterally increased DPFC (BA9/46) activity during rule discovery but increased VPFC (BA47/12) activity with changes in card-sorting rule on the WCST (Monchi et al. 2001). It is possible that SGAs have different effects on dorsal and ventral prefrontal brain regions.

The observation that in this study, working memory task performance did not differ between the two treatment groups, is noteworthy given that there were differences between the two groups at the level of BOLD in response to working memory load increase. Dissociations of effects at behavioural and neural levels of analysis in fMRI designs have previously been described in relation to experimental cognitive neuroscience (Wilkinson and Halligan 2004), but similar arguments may apply to pharmacological fMRI studies (Migo et al. 2011). Specifically, in this instance, it may be argued that our data provide evidence for the notion that the BOLD signal may be a more sensitive measure of pharmacological treatment effects than behavioural measures (Honey and Bullmore 2004) analogous to findings in the neurochemical imaging literature (Fannon et al. 2003).

A likely pharmacological explanation of the treatment effects in this study may be the richer pharmacological profile of SGA compounds which rely not just on D2 receptor antagonism but also act on other neurotransmitter systems such as the serotonergic system. However, the group of SGA compounds used in this study is heterogeneous, thus not allowing a definitive explanation of the neurotransmitter mechanisms underlying the effects observed here.

\section{Limitations}

This study has a number of limitations. First, the patient group treated with FGA was small $(N=6)$. This limitation of the study stems from the fact that we used a naturalistic design in a clinical environment where the majority of schizophrenia patients are treated with SGA. The small size of the FGA group could also be an explanation for the lack of differences between the two patient groups in demo- 
graphic, clinical, or performance measures. An additional limitation is that the group of SGA is pharmacologically heterogeneous. Therefore, it cannot be concluded that there is a single mechanism common to all these compounds by which they affected BOLD in this study. It should also be noted that the performance-by-load and treatment-by-load interactions were observed only when considering individual ROIs but not at the voxelwise level. Previous studies have noted that antipsychotic effects at the level of BOLD may be of small to moderate effect size (Meisenzahl et al. 2006); therefore, replication using larger samples will be important. A further limitation is the fact that some patients were co-medicated with other compounds; these may influence cognition and brain function. Finally, the effects of pharmacological treatment should ideally be investigated using more powerful longitudinal designs rather than crosssectional studies. Future longitudinal studies are needed to further advance this field.

Acknowledgements We extend our gratitude to the patients and controls who gave their time to participate in this study. We would like to thank Susanne Karch, $\mathrm{PhD}$, for helpful discussion of these data. This work was funded by the Deutsche Forschungsgemeinschaft (ET 31/2-1) and the Wellcome Trust (067427/z/02).

Statement of interest U. Ettinger, S. Williams, D. Fannon, P. Premkumar, E. Kuipers, and V. Kumari have no interest to declare. H.J. Möller has received grants or is a consultant for and on the speakership bureaus of AstraZeneca, Bristol-Myers Squibb, Eisai, Eli Lilly, GlaxoSmithKline, Janssen Cilag, Lundbeck, Merck, Novartis, Organon, Pfizer, Sanofi-Aventis, Schering-Plough, Schwabe, Sepracor, Servier and Wyeth. None of these companies were involved in any aspect of this study.

Open Access This article is distributed under the terms of the Creative Commons Attribution Noncommercial License which permits any noncommercial use, distribution, and reproduction in any medium, provided the original author(s) and source are credited.

\section{References}

American Psychiatric Association (1994) Diagnostic and statistical manual of mental disorders, fourth edition (DSM-IV). American Psychiatric Association, Washington

Barch DM (2005) The cognitive neuroscience of schizophrenia. Annu Rev Clin Psychol 1:321-353

Barch DM, Smith E (2008) The cognitive neuroscience of working memory: relevance to CNTRICS and schizophrenia. Biol Psychiatry 64:11-17

Braus DF, Ende G, Weber-Fahr W, Sartorius A, Krier A, HubrichUngureanu P, Ruf M, Stuck S, Henn FA (1999) Antipsychotic drug effects on motor activation measured by functional magnetic resonance imaging in schizophrenic patients. Schizophr Res 39:19-29

Brett M, Anton JL, Valabregue R, Poline JB (2002) Region of interest analysis using an SPM toolbox [abstract]. Presented at the 8th International Conference on Functional Mapping of the Human Brain, June 2-6, 2002, Sendai, Japan. Neuroimage 15
Callicott JH, Mattay VS, Verchinski BA, Marenco S, Egan MF, Weinberger DR (2003) Complexity of prefrontal cortical dysfunction in schizophrenia: more than up or down. Am J Psychiatry 160:2209-2215

Davis CE, Jeste DV, Eyler LT (2005) Review of longitudinal functional neuroimaging studies of drug treatments in patients with schizophrenia. Schizophr Res 78:45-60

Egan MF, Goldberg TE, Kolachana BS, Callicott JH, Mazzanti CM, Straub RE, Goldman D, Weinberger DR (2001) Effect of COMT Val108/158 Met genotype on frontal lobe function and risk for schizophrenia. Proc Natl Acad Sci USA 98:6917-6922

Fannon D, Simmons A, Tennakoon L, O'Ceallaigh S, Sumich A, Doku V, Shew C, Sharma T (2003) Selective deficit of hippocampal $\mathrm{N}$-acetylaspartate in antipsychotic-naive patients with schizophrenia. Biol Psychiatry 54:587-598

First MB, Spitzer RL, Gibbon M, Williams JBW (1996) Structured clinical interview for DSM-IV axis I disorders, clinician version (SCID-CV). American Psychiatric Press Inc, Washington

Glahn DC, Ragland JD, Abramoff A, Barrett J, Laird AR, Bearden CE, Velligan DI (2005) Beyond hypofrontality: a quantitative meta-analysis of functional neuroimaging studies of working memory in schizophrenia. Hum Brain Mapp 25:60-69

Goldman-Rakic PS (1994) Working memory dysfunction in schizophrenia. J Neuropsychiatry Clin Neurosci 6:348-357

Green MF (2001) Schizophrenia revealed. WW Norton, New York

Green MF, Nuechterlein KH (2004) The MATRICS initiative: developing a consensus cognitive battery for clinical trials. Schizophr Res 72:1-3

Greenwood KE, Landau S, Wykes T (2005) Negative symptoms and specific cognitive impairments as combined targets for improved functional outcome within cognitive remediation therapy. Schizophr Bull 31:910-921

Gruber O, von Cramon DY (2003) The functional neuroanatomy of human working memory revisited. Evidence from 3-T fMRI studies using classical domain-specific interference tasks. Neuroimage 19:797-809

Henseler I, Gruber O, Kraft S, Krick C, Reith W, Falkai P (2008) Compensatory hyperactivations as markers of latent working memory dysfunctions in patients with obsessive-compulsive disorder: an fMRI study. J Psychiatry Neurosci 33:209-215

Honey G, Bullmore E (2004) Human pharmacological MRI. Trends Pharmacol Sci 25:366-374

Honey GD, Bullmore ET, Soni W, Varatheesan M, Williams SC, Sharma T (1999) Differences in frontal cortical activation by a working memory task after substitution of risperidone for typical antipsychotic drugs in patients with schizophrenia. Proc Natl Acad Sci U S A 96:13432-13437

Jones HM, Brammer MJ, O'Toole M, Taylor T, Ohlsen RI, Brown RG, Purvis R, Williams S, Pilowsky LS (2004) Cortical effects of quetiapine in first-episode schizophrenia: a preliminary functional magnetic resonance imaging study. Biol Psychiatry 56:938-942

Karch S, Leicht G, Giegling I, Lutz J, Kunz J, Buselmeier M, Hey P, Sporl A, Jager L, Meindl T, Pogarell O, Moller HJ, Hegerl U, Rujescu D, Mulert C (2009) Inefficient neural activity in patients with schizophrenia and nonpsychotic relatives of schizophrenic patients: evidence from a working memory task. J Psychiatr Res 43:1185-1194

Kay SR, Fiszbein A, Opler LA (1987) The positive and negative syndrome scale (PANSS) for schizophrenia. Schizophr Bull $13: 261-276$

Kumari V, Cooke M (2006) Use of magnetic resonance imaging in tracking the course and treatment of schizophrenia. Expert Rev Neurother 6:1005-1016

Kumari V, Antonova E, Geyer MA, ffytche D, Williams SC, Sharma T (2007) A fMRI investigation of startle gating deficits in 
schizophrenia patients treated with typical or atypical antipsychotics. Int J Neuropsychopharmacol 10:463-477

Kumari V, Peters ER, Fannon D, Antonova E, Premkumar P, Anilkumar AP, Williams SC, Kuipers E (2009) Dorsolateral prefrontal cortex activity predicts responsiveness to cognitivebehavioral therapy in schizophrenia. Biol Psychiatry 66:594-602

Lee J, Folley BS, Gore J, Park S (2008) Origins of spatial working memory deficits in schizophrenia: an event-related FMRI and near-infrared spectroscopy study. PLoS One 3:e1760

Liddle PF (2000) Cognitive impairment in schizophrenia: its impact on social functioning. Acta Psychiatr Scand Suppl 400:11-16

Linden D (2009) Towards a functional neuroanatomy of symptoms and cognitive deficits of schizophrenia. In: Ritsner MS (ed) The handbook of neuropsychiatric biomarkers, endophenotypes and genes. Springer, Heidelberg, p 31

Manoach DS (2003) Prefrontal cortex dysfunction during working memory performance in schizophrenia: reconciling discrepant findings. Schizophr Res 60:285-298

Meisenzahl EM, Scheuerecker J, Zipse M, Ufer S, Wiesmann M, Frodl T, Koutsouleris N, Zetzsche T, Schmitt G, Riedel M, Spellmann I, Dehning S, Linn J, Bruckmann H, Moller HJ (2006) Effects of treatment with the atypical neuroleptic quetiapine on working memory function: a functional MRI follow-up investigation. Eur Arch Psychiatry Clin Neurosci 256:522-531

Meyer-Lindenberg A, Weinberger DR (2006) Intermediate phenotypes and genetic mechanisms of psychiatric disorders. Nat Rev Neurosci 7:818-827

Migo E, Williams SCR, Crum W, Kempton M, Ettinger U (2011) The role of neuroimaging biomarkers in personalised medicine for neuro-degenerative and psychiatric disorders. In: Gordon E, Koslow SH (eds) Integrative neuroscience and personalized medicine, Chapter 3. Oxford University Press, pp 141-163

Minzenberg MJ, Laird AR, Thelen S, Carter CS, Glahn DC (2009) Meta-analysis of 41 functional neuroimaging studies of executive function in schizophrenia. Arch Gen Psychiatry 66:811-822

Monchi O, Petrides M, Petre V, Worsley K, Dagher A (2001) Wisconsin Card Sorting revisited: distinct neural circuits participating in different stages of the task identified by event-related functional magnetic resonance imaging. J Neurosci 21:7733-7741

Park S, Holzman PS (1992) Schizophrenics show spatial working memory deficits. Arch Gen Psychiatry 49:975-982
Potkin SG, Turner JA, Brown GG, McCarthy G, Greve DN, Glover GH, Manoach DS, Belger A, Diaz M, Wible CG, Ford JM, Mathalon DH, Gollub R, Lauriello J, O'Leary D, van Erp TG, Toga AW, Preda A, Lim KO (2009) Working memory and DLPFC inefficiency in schizophrenia: the FBIRN study. Schizophr Bull 35:19-31

Royer A, Schneider FC, Grosselin A, Pellet J, Barral FG, Laurent B, Brouillet D, Lang F (2009) Brain activation during executive processes in schizophrenia. Psychiatry Res 173:170-176

Schlagenhauf F, Wustenberg T, Schmack K, Dinges M, Wrase J, Koslowski M, Kienast T, Bauer M, Gallinat J, Juckel G, Heinz A (2008) Switching schizophrenia patients from typical neuroleptics to olanzapine: effects on BOLD response during attention and working memory. Eur Neuropsychopharmacol 18:589-599

Schneider F, Habel U, Reske M, Kellermann T, Stocker T, Shah NJ, Zilles K, Braus DF, Schmitt A, Schlosser R, Wagner M, Frommann I, Kircher T, Rapp A, Meisenzahl E, Ufer S, Ruhrmann S, Thienel R, Sauer H, Henn FA, Gaebel W (2007) Neural correlates of working memory dysfunction in first-episode schizophrenia patients: an fMRI multi-center study. Schizophr Res 89:198-210

Stephan KE, Magnotta VA, White T, Arndt S, Flaum M, O'Leary DS, Andreasen NC (2001) Effects of olanzapine on cerebellar functional connectivity in schizophrenia measured by fMRI during a simple motor task. Psychol Med 31:1065-1078

Surguladze SA, Chu EM, Evans A, Anilkumar AP, Patel MX, Timehin C, David AS (2007) The effect of long-acting risperidone on working memory in schizophrenia: a functional magnetic resonance imaging study. J Clin Psychopharmacol 27: $560-570$

Talairach J, Tournoux P (1988) Co-planar stereotaxic atlas of the human brain. Thieme Medical Publishers, New York

Tan HY, Choo WC, Fones CS, Chee MW (2005) fMRI study of maintenance and manipulation processes within working memory in first-episode schizophrenia. Am J Psychiatry 162:1849-1858

van Raalten TR, Ramsey NF, Jansma JM, Jager G, Kahn RS (2008) Automatization and working memory capacity in schizophrenia. Schizophr Res 100:161-171

Wilkinson D, Halligan P (2004) The relevance of behavioural measures for functional-imaging studies of cognition. Nat Rev Neurosci 5:67-73 